\title{
Safety profile of statins alone or combined with ezetimibe: a pooled analysis of 27 studies including over 22,000 patients treated for 6-24 weeks
}

\author{
P. P. Toth, ${ }^{1}$ D. Morrone, ${ }^{2}$ W. S. Weintraub, ${ }^{2}$ M. E. Hanson, ${ }^{3}$ R. S. Lowe, ${ }^{3}$ J. Lin, ${ }^{4}$ A. K. Shah, ${ }^{4}$ \\ A. M. Tershakovec ${ }^{5}$
}

${ }^{1}$ CGH Medical Center, Sterling, $\mathrm{L}$, and University of Illinois College of Medicine, Peoria, IL, USA

${ }^{2}$ Christiana Center for

Outcomes Research, Christiana Care Health System, Newark, $D E$, USA

${ }^{3}$ Global Scientific and Medical Publications, Merck Sharp \& Dohme Corp., Whitehouse Station, NJ, USA

${ }^{4}$ Clinical and Quantitative Sciences, Merck Sharp \& Dohme Corp., Whitehouse Station, NJ, USA

${ }^{5}$ Project Leadership and Management, Merck Sharp \& Dohme Corp., Whitehouse Station, NJ, USA

Correspondence to: Peter P.

Toth, M.D., Ph.D.

Director of Preventive

Cardiology, CGH Medical

Center, Sterling, IL and Clinical Professor, University of Illinois School of Medicine, Peoria, IL, USA

Tel.: (815) 632-5093 Fax: (815) 626-5947

Email: peter.toth@cghmc.com

Disclosure

Dr. Toth: Acted as a consultant for Abbott Laboratories, AstraZeneca, Atherotech, Kowa and Merck \& Co. Inc; served on speakers' bureaus for Abbott Laboratories, Amylin,

AstraZeneca, Atherotech, Boehringer-Ingelheim, Glaxo Smith Kline, Kowa, Merck \& Co. Inc; Drs. Morrone and Weintraub: No conflicts of interest; Drs. Hanson, Lowe, Shah and Tershakovec and Mr. Lin are employees of Merck

Sharp \& Dohme Corp. and may own stock or hold stock options in the company.

\section{SUMMARY}

Aims: The aim of this analysis was to assess the overall safety and tolerability profiles of various statins + ezetimibe vs. statin monotherapy and to explore tolerability in sub-populations grouped by age, race, and sex. Methods: Study-level data were combined from 27 double-blind, placebo-controlled or active-comparator trials that randomized adult hypercholesterolemic patients to statin or statin + ezetimibe for 6-24 weeks. In the full cohort, \% patients with AEs within treatment groups (statin: $N=10,517$; statin + ezetimibe: $N=11,714$ ) was assessed by logistic regression with terms for first-/second-line therapy (first line = drug-naïve or rendered drug-naïve by washout at study entry; second line = ongoing statin at study entry or statin run-in), trial within first-/second-line therapy, and treatment. The same model was fitted for age $(<65, \geq 65$ years), sex, race (white, black, other) and first-/second-line subgroups with additional terms for subgroup and subgroup-by-treatment interaction. Results: In the full cohort, the only significant difference between treatments was consecutive AST or ALT elevations $\geq 3 \times$ upper limit of normal (ULN) (statin: $0.35 \%$, statin + ezetimibe: $0.56 \% ; p=0.017$ ). Significantly more subjects reported $\geq 1 \mathrm{AE}$; drug-related, hepatitis-related and gastrointestinal-related AEs; and CK elevations $\geq 10 \times$ ULN (all $p \leq 0.008$ ) in first-line vs. second-line therapy studies with both treatments. AEs were generally similar between treatments in subgroups, and similar rates of AEs were reported within age and race subgroups; however, women reported generally higher AE rates. Conclusions: In conclusion, in second-line studies, ongoing statin treatment at study entry likely screened out participants for previous statin-related AEs and tolerability issues. These results describe the safety profiles of widely used lipid-lowering therapies and encourage their appropriate and judicious use in certain subpopulations.

\section{Introduction}

The National Cholesterol Education Program Adult Treatment Panel (NCEP ATP) III guidelines recommend a low-density lipoprotein cholesterol (LDL-C) goal $<100 \mathrm{mg} / \mathrm{dl} \quad(2.59 \mathrm{mmol} / \mathrm{l})$ for high-risk patients (those with clinical cardiovascular disease, diabetes, or 10-year coronary heart disease [CHD] risk $>20 \%$ ) and suggest an optional LDL-C goal $<70 \mathrm{mg} / \mathrm{dl}(1.81 \mathrm{mmol} / \mathrm{l})$ for those at the highest risk, including those with established cardiovascular disease plus additional high-risk characteristics such as diabetes mellitus, multiple cardiovascular risk fac-

\section{What's known}

The general safety and tolerability profiles of statin monotherapy and statin + ezetimibe combination therapy have been established. Comparable safety and tolerability profiles have been reported in subgroups by sex, race and age, although these secondary analyses have not been powered to assess statistical differences. Some cases of myopathy and rare cases of rhabdomyolysis have been reported during treatment with statins and ezetimibe.

\section{What's new}

The general safety and tolerability profiles of statin monotherapy and statin + ezetimibe combination therapy were confirmed. Irrespective of treatment group, generally higher rates of AEs were reported in women vs. men, and generally similar rates of AEs were reported between races and between age groups. More AEs were reported in first-line studies vs. second-line studies. The occurrence of myopathy was low and did not differ between treatments or any of the subgroups studied. 
rienced with high doses of single agents. The potential disadvantages of combination therapy include drug interactions, increased number of pills, greater cost, and additive side effects. Moreover, data on clinical outcomes with combination therapy are limited.

The general safety and tolerability profiles of statin monotherapy and statin + ezetimibe combination therapy have been established in multiple clinical trials [reviewed in Refs $(3,4)$ ]. Cases of myopathy and rare cases of rhabdomyolysis have been reported during treatment with statins (5) and ezetimibe (6). With statins, these cases can occur at any dose level, but the risks increase at the higher doses and with certain concomitant medications (7-11). Clinical trials that have assessed lipid-lowering treatments are generally not powered to identify small betweentreatment differences in safety and tolerability or rare adverse experiences. Moreover, conclusions drawn about differences in subpopulations, such as those assessed by age, race and sex, have been limited due to group size. The objective of this analysis was to assess the overall safety and tolerability profiles of various statins + ezetimibe vs. statin monotherapy and to explore tolerability in sub-populations grouped by age, race, and sex in a population of over 22,000 patients using pooled data combined from previously published trials.

\section{Methods}

In this analysis, study-level data were combined from 27 double-blind, placebo-controlled or activecomparator studies conducted between 1999 and 2008 by Merck Research Laboratories. In these studies adult hypercholesterolemic patients were randomized to statin or statin + ezetimibe for 6-24 weeks (Table 1) with a mean follow up duration of 9 weeks. Included studies were relatively short-term, lipid-lowering trials. Studies with cross-over design, extension studies, studies still ongoing, imaging studies, studies in which ezetimibe was used as monotherapy or in combination with other non-statin lipid-lowering drugs (e.g., fenofibrate, niacin), adolescent or pediatric patient studies, and studies focusing on patients with sitosterolemia, homozygous familial hypercholesterolemia, aortic stenosis, or chronic kidney disease were not included in these analyses.

Specific inclusion criteria for the individual studies have been previously published (see citations in Table 1). Since guidelines have changed over time, there was no single lipid entry criterion that applied to all studies. Generally, a patient was considered hypercholesterolemic if LDL-C levels were above guideline-recommended levels according to risk. The range of baseline LDL-C inclusion levels in the stud- ies was between $70 \mathrm{mg} / \mathrm{dl} \quad(1.81 \mathrm{mmol} / \mathrm{l})$ to $250 \mathrm{mg} / \mathrm{dl}$ (6.47 mmol/l; Table 1).

Thirteen studies evaluated first-line therapy, which included subjects who were drug-naïve or rendered drug-naïve by wash-out at study start, and randomized patients to receive double-blind ezetimibe/statin [ezetimibe/simvastatin combination tablet (10/10, $10 / 20,10 / 40$, or $10 / 80 \mathrm{mg}$ ) or ezetimibe $10 \mathrm{mg} \mathrm{co-}$ administered with: simvastatin $10,20,40$, or $80 \mathrm{mg}$; lovastatin 10,20 , or $40 \mathrm{mg}$; pravastatin 10,20 , or $40 \mathrm{mg}$ or atorvastatin $10,20,40$ or $80 \mathrm{mg}$ ] or statin alone (simvastatin 10, 20, 40, and $80 \mathrm{mg}$; lovastatin 10,20 , and $40 \mathrm{mg}$; pravastatin 10,20 , and $40 \mathrm{mg}$; atorvastatin $10,20,40$, and $80 \mathrm{mg}$ or rosuvastatin 10 , 20 , or $40 \mathrm{mg}$ ) for up to 12 weeks. There were 14 studies that evaluated second-line therapy, which included subjects who were previously on statins or receiving statins during run-in. In the add-on therapy studies (11 studies), statin-treated patients were randomized to receive double-blind placebo or ezetimibe $10 \mathrm{mg}$ administered in combination with their ongoing, previously prescribed, open-label statin, which may have been doubled in some studies (simvastatin 10, 20, 40, or $80 \mathrm{mg}$; lovastatin 10,20 , or $40 \mathrm{mg}$; pravastatin 10 , 20 , or $40 \mathrm{mg}$; fluvastatin 20,40,80, or $160 \mathrm{mg}$; atorvastatin $10,20,40$, or $80 \mathrm{mg}$ or cerivastatin $0.2,0.3$, 0.4 , or $0.8 \mathrm{mg}$ ) for up to 8 weeks. In "switch-therapy" studies (3 studies), statin-treated patients were switched from their ongoing, previously prescribed, open-label statin (simvastatin 10,20 , or $40 \mathrm{mg}$; lovastatin 10 or $20 \mathrm{mg}$; pravastatin 10 or $20 \mathrm{mg}$; fluvastatin 10 or $40 \mathrm{mg}$; atorvastatin 10,20 , or $40 \mathrm{mg}$ or rosuvastatin $5 \mathrm{mg}$ ) to receive double-blind ezetimibe/statin (ezetimibe/simvastatin combination tablet $10 / 20$ or $10 / 40 \mathrm{mg}$ ) or statin alone (simvastatin 20 , 40 , or $80 \mathrm{mg}$; lovastatin 20 or $40 \mathrm{mg}$; pravastatin 20 or $40 \mathrm{mg}$; fluvastatin 20 or $80 \mathrm{mg}$; atorvastatin 20, 40, or $80 \mathrm{mg}$ or rosuvastatin $10 \mathrm{mg}$ ) for up to 24 weeks. Specific statin type and dose were matched between comparison groups in some trials, and in other trials, common-use comparisons were made (i.e., doses were compared that would frequently be used in a clinical setting, e.g. starting dose vs. starting dose, next higher dose vs. next-higher dose; Table 1).

Adverse experiences (AEs) were summarized by system organ class and specific adverse experience term. Prespecified safety parameters included alanine aminotransferase (ALT) and/or aspartate aminotransferase (AST) consecutive elevations $\geq 3 \times$ upper limit of normal (ULN); creatine kinase (CK) elevations $\geq 10 \times \mathrm{ULN}$; CK elevations $\geq 10 \times \mathrm{ULN}$ with muscle symptoms (myopathy), and myopathy with associated evidence of renal damage (rhabdomyolysis). Special AEs of interest included hepatitisrelated, gallbladder-related, gastrointestinal-related, 
Table 1 Characteristics of studies included in the combined analyses

\begin{tabular}{|c|c|c|c|c|c|c|}
\hline \multirow{2}{*}{$\begin{array}{l}\text { Protocol } \\
\text { number } \\
\text { (citation) }\end{array}$} & \multirow[b]{2}{*}{ Treatment } & \multirow{2}{*}{$\begin{array}{l}\text { Study } \\
\text { duration }\end{array}$} & \multirow{2}{*}{$\begin{array}{l}n \text { of subjects } \\
\text { who took statin } \\
(N=10,387)\end{array}$} & \multirow{2}{*}{$\begin{array}{l}n \text { of subjects } \\
\text { who took statin }+E Z \\
(N=11,891)\end{array}$} & \multicolumn{2}{|l|}{ Inclusion criteria } \\
\hline & & & & & Min LDL-C & Max LDL-C \\
\hline 005 (24) & $\begin{array}{l}E Z, P B O \\
E Z+S 20,40,80 \\
S 20,40,80\end{array}$ & 12 weeks & 229 & 539 & $145 \mathrm{mg} / \mathrm{dl}(3.75 \mathrm{mmol} / \mathrm{l})$ & $250 \mathrm{mg} / \mathrm{dl}(6.47 \mathrm{mmol} / \mathrm{l})$ \\
\hline $011(25)$ & $\begin{array}{l}E Z, P B O \\
S 10,20,40,80 \\
E Z+S 10,20,40,80\end{array}$ & 12 weeks & 263 & 274 & $145 \mathrm{mg} / \mathrm{dl}(3.75 \mathrm{mmol} / \mathrm{l})$ & $250 \mathrm{mg} / \mathrm{dl}(6.47 \mathrm{mmol} / \mathrm{l})$ \\
\hline $021(26)$ & $\begin{array}{l}E Z+S 20 \\
S 40\end{array}$ & 24 weeks & 110 & 104 & $101 \mathrm{mg} / \mathrm{dl}(2.62 \mathrm{mmol} / \mathrm{l})$ & Not specified \\
\hline $023(27)$ & $\begin{array}{l}S 20 \\
E Z+S 20,40,80\end{array}$ & 23 weeks & 253 & 457 & $130 \mathrm{mg} / \mathrm{dl}(3.37 \mathrm{mmol} / \mathrm{l})$ & Not specified \\
\hline $025(28)$ & $\begin{array}{l}\text { A } 10-80 \\
\text { EZ/S } 10-80\end{array}$ & 24 weeks & 262 & 526 & Not at LDL-C goal as define & ICEP ATP III \\
\hline $030(29)$ & $\begin{array}{l}E Z+A 10,20,40 \\
A 10,20,40,80\end{array}$ & 14 weeks & 316 & 305 & $130 \mathrm{mg} / \mathrm{dl}(3.37 \mathrm{mmol} / \mathrm{l})$ & Not specified \\
\hline $038(30)$ & $\begin{array}{l}\text { EZ, PBO } \\
\text { EZ/S 10, 20,40,80 } \\
\text { S } 10,20,40,80\end{array}$ & 12 weeks & 622 & 609 & $145 \mathrm{mg} / \mathrm{dl}(3.75 \mathrm{mmol} / \mathrm{l})$ & $250 \mathrm{mg} / \mathrm{dl}(6.47 \mathrm{mmol} / \mathrm{l})$ \\
\hline $040(31)$ & $\begin{array}{l}\text { EZ + ongoing statin } \\
\text { Ongoing statin }\end{array}$ & 6 weeks & 1009 & 2009 & Not at LDL-C goal as define & ICEP ATP III \\
\hline 051 (32) & $\begin{array}{l}E Z / S 20,40,80 \\
\text { A } 1020,40,80\end{array}$ & 6 weeks & 947 & 948 & Not at LDL-C goal as define & ICEP ATP III \\
\hline $058(33)$ & $\begin{array}{l}E Z / S 20,40,80 \\
\text { R } 10,20,40\end{array}$ & 6 weeks & 1477 & 1474 & $145 \mathrm{mg} / \mathrm{dl}(3.75 \mathrm{mmol} / \mathrm{l})$ & $250 \mathrm{mg} / \mathrm{dl}(6.47 \mathrm{mmol} / \mathrm{l})$ \\
\hline 077 (34) & $\begin{array}{l}E Z / S 20,40 \\
\text { A } 10,20,40\end{array}$ & 6 weeks & 732 & 494 & $100 \mathrm{mg} / \mathrm{dl}(2.59 \mathrm{mmol} / \mathrm{l})$ & Not specified \\
\hline 079 (35) & $\begin{array}{l}\mathrm{EZ}+\mathrm{A} 20 \\
\mathrm{~A} 40\end{array}$ & 6 weeks & 98 & 96 & $100 \mathrm{mg} / \mathrm{dl}(2.59 \mathrm{mmol} / \mathrm{l})$ & $160 \mathrm{mg} / \mathrm{dl}(4.14 \mathrm{mmol} / \mathrm{l})$ \\
\hline $090(36)$ & $\begin{array}{l}E Z+A 40 \\
A 80\end{array}$ & 6 weeks & 289 & 286 & $70 \mathrm{mg} / \mathrm{dl}(1.81 \mathrm{mmol} / \mathrm{l})$ & $160 \mathrm{mg} / \mathrm{dl}(4.14 \mathrm{mmo} / \mathrm{l})$ \\
\hline $107(37)$ & $\begin{array}{l}E Z / S 20,40 \\
\text { A } 10,20,40\end{array}$ & 6 weeks & 678 & 450 & $\begin{array}{l}70 \mathrm{mg} / \mathrm{dl}(1.81 \mathrm{mmol} / \mathrm{l}) \\
100 \mathrm{mg} / \mathrm{dl}(2.59 \mathrm{mmol} / \mathrm{l})\end{array}$ & Not specified \\
\hline $112(38)$ & $\begin{array}{l}E Z+A 10 \\
A 20 / 40\end{array}$ & 6 weeks & 525 & 526 & 70 mg/dl $(1.81 \mathrm{mmol} / \mathrm{l})$ & $160 \mathrm{mg} / \mathrm{dl}(4.14 \mathrm{mmol} / \mathrm{l})$ \\
\hline 679 (39) & $\begin{array}{l}\text { EZ, PBO } \\
\text { L } 10,20,40 \\
E Z+L 10,20,40\end{array}$ & 12 weeks & 220 & 192 & $145 \mathrm{mg} / \mathrm{dl}(3.75 \mathrm{mmol} / \mathrm{l})$ & $250 \mathrm{mg} / \mathrm{dl}(6.47 \mathrm{mmol} / \mathrm{l})$ \\
\hline $691(40)$ & $\begin{array}{l}\text { EZ, PBO } \\
\text { P } 10,20,40 \\
\text { EZ }+ \text { P 10, 20, } 40\end{array}$ & 12 weeks & 205 & 204 & $145 \mathrm{mg} / \mathrm{dl}(3.75 \mathrm{mmol} / \mathrm{l})$ & $250 \mathrm{mg} / \mathrm{dl}(6.47 \mathrm{mmol} / \mathrm{l})$ \\
\hline $692(41)$ & $\begin{array}{l}\text { EZ, PBO } \\
\text { A } 10,20,40,80 \\
\text { EZ + A 10, 20, 40, } 80\end{array}$ & 12 weeks & 248 & 255 & $145 \mathrm{mg} / \mathrm{dl}(3.75 \mathrm{mmol} / \mathrm{l})$ & $250 \mathrm{mg} / \mathrm{dl}(6.47 \mathrm{mmol} / \mathrm{l})$ \\
\hline $700(42)$ & $\begin{array}{l}\text { EZ } \\
\text { S } 20,40\end{array}$ & 14 weeks & 34 & 66 & $130 \mathrm{mg} / \mathrm{dl}(3.37 \mathrm{mmol} / \mathrm{l})$ & Not specified \\
\hline $801(43)$ & $\begin{array}{l}E Z+S 10,20 \\
S 10,20\end{array}$ & 6 weeks & 210 & 208 & $101 \mathrm{mg} / \mathrm{dl}(2.62 \mathrm{mmol} / \mathrm{l})$ & $160 \mathrm{mg} / \mathrm{dl}(4.14 \mathrm{mmo} / \mathrm{l})$ \\
\hline $802(44)$ & $\begin{array}{l}E Z+S 10,20 \\
S 10,20\end{array}$ & 6 weeks & 191 & 181 & $101 \mathrm{mg} / \mathrm{dl}(2.62 \mathrm{mmol} / \mathrm{l})$ & $160 \mathrm{mg} / \mathrm{dl}(4.14 \mathrm{mmol} / \mathrm{l})$ \\
\hline 803/804 (45) & $\begin{array}{l}E Z+A 10,20 \\
A 10,20\end{array}$ & 6 weeks & 230 & 220 & $101 \mathrm{mg} / \mathrm{dl}(2.62 \mathrm{mmol} / \mathrm{l})$ & $160 \mathrm{mg} / \mathrm{dl}(4.14 \mathrm{mmol} / \mathrm{l})$ \\
\hline $806(46)$ & $\begin{array}{l}\mathrm{EZ} / \mathrm{S} 20 \\
\mathrm{~A} 20\end{array}$ & 6 weeks & 214 & 221 & $100 \mathrm{mg} / \mathrm{dl}(2.62 \mathrm{mmol} / \mathrm{l})$ & $160 \mathrm{mg} / \mathrm{dl}(4.14 \mathrm{mmol} / \mathrm{l})$ \\
\hline $807(47)$ & $\begin{array}{l}\text { ES } 20,40 \\
\text { A } 20\end{array}$ & 6 weeks & 219 & 442 & $101 \mathrm{mg} / \mathrm{dl}(2.62 \mathrm{mmol} / \mathrm{l})$ & $160 \mathrm{mg} / \mathrm{dl}(4.14 \mathrm{mmol} / \mathrm{l})$ \\
\hline $809(48)$ & $\begin{array}{l}\mathrm{EZ} / \mathrm{S} 20 \\
\mathrm{R} 10\end{array}$ & 6 weeks & 293 & 302 & $100 \mathrm{mg} / \mathrm{dl}(2.59 \mathrm{mmol} / \mathrm{l})$ & $160 \mathrm{mg} / \mathrm{dl}(4.14 \mathrm{mmol} / \mathrm{l})$ \\
\hline 2173/2246 (49) & $\begin{array}{l}\text { EZ + ongoing statin } \\
\text { Ongoing statin }\end{array}$ & 8 weeks & 390 & 379 & Not at LDL-C goal as define & ICEP ATP III \\
\hline $3377(50)$ & $\begin{array}{l}E Z+S 20 \\
S 20\end{array}$ & 12 weeks & 123 & 124 & $145 \mathrm{mg} / \mathrm{dl}(3.75 \mathrm{mmol} / \mathrm{l})$ & $250 \mathrm{mg} / \mathrm{dl}(6.47 \mathrm{mmol} / \mathrm{l})$ \\
\hline
\end{tabular}

A, atorvastatin; EZ, ezetimibe; L, lovastatin; LDL-C, low-density lipoprotein cholesterol; NCEP ATP III, National Cholesterol Education Program Adult Treatment Panel III; P, pravastatin; R, rosuvastatin; S, simvastatin. 
Table 2 Baseline characteristics for ITT population and subgroups included in analyses

ITT population

\begin{tabular}{|c|c|c|c|c|}
\hline Parameter & \multicolumn{2}{|c|}{$\begin{array}{l}\text { Statin } \\
(N=10,308)\end{array}$} & $\begin{array}{l}\text { Statin/EZ } \\
(N=11,486)\end{array}$ & $\begin{array}{l}\text { Total } \\
(N=21,794)\end{array}$ \\
\hline Male, $n(\%)$ & \multicolumn{2}{|c|}{$5279(51.2)$} & $6016(52.4)$ & $11,295(51.8)$ \\
\hline Female, $n(\%)$ & \multicolumn{2}{|c|}{$5029(48.8)$} & $5470(47.6)$ & $10,499(48.2)$ \\
\hline Mean age, years (SD) & \multicolumn{2}{|c|}{$59.2(11.3)$} & $59.9(11.1)$ & $59.6(11.2)$ \\
\hline$<65$ years, $n(\%)$ & \multicolumn{2}{|c|}{$6682(64.8)$} & $7250(63.1)$ & $13,932(63.9)$ \\
\hline$\geq 65$ years, $n(\%)$ & \multicolumn{2}{|c|}{$3626(35.2)$} & $4236(36.9)$ & 5989 (36.1) \\
\hline Caucasian, $n(\%)$ & \multicolumn{2}{|c|}{$8690(84.3)$} & $9706(84.5)$ & $18,396(84.4)$ \\
\hline Non-Caucasian, $n(\%)$ & \multicolumn{2}{|c|}{$1618(15.7)$} & $1780(15.5)$ & 3398 (15.6) \\
\hline CHD, $n(\%)$ & \multicolumn{2}{|c|}{$3310(32.1)$} & 4115 (35.9) & $7425(34.1)$ \\
\hline Diabetes mellitus, $n(\%)$ & \multicolumn{2}{|c|}{$3082(29.9)$} & 3459 (30.1) & $6541(30.0)$ \\
\hline Metabolic syndrome, $n(\%)$ & \multicolumn{2}{|c|}{$4233(50.7)$} & $4518(49.7)$ & $8751(50.1)$ \\
\hline First-line, $n(\%)$ & \multicolumn{2}{|c|}{$6257(60.7)$} & $6241(54.3)$ & $12,498(57.3)$ \\
\hline Second-line, $n(\%)$ & 4051 & & $5245(45.7)$ & $9296(42.7)$ \\
\hline $\mathrm{BMI} \geq 30 \mathrm{~kg} / \mathrm{m}^{2}, n(\%)$ & 4138 & & $4537(40.1)$ & $8675(40.4)$ \\
\hline BMI $\left(\mathrm{kg} / \mathrm{m}^{2}\right)$ & $(n=1$ & & $(n=11,315)$ & $(n=21,458)$ \\
\hline Mean (SD) & 29.8 & & $29.7(5.8)$ & $29.8(5.8)$ \\
\hline & & & & \\
\hline & $\begin{array}{l}\text { Statin } \\
(n=5279)\end{array}$ & $\begin{array}{l}\text { Statin/EZ } \\
(n=6016)\end{array}$ & $\begin{array}{l}\text { Statin } \\
(n=5029)\end{array}$ & $\begin{array}{l}\text { Statin/EZ } \\
(n=5470)\end{array}$ \\
\hline Mean age, years (SD) & $58.4(11.2)$ & $59.2(11.1)$ & $60.0(11.2)$ & $60.7(11.0)$ \\
\hline$<65$ years, $n(\%)$ & $3556(67.4)$ & $3909(65.0)$ & $3126(62.2)$ & $3341(61.1)$ \\
\hline$\geq 65$ years, $n(\%)$ & $1723(32.6)$ & $2107(35.0)$ & $1903(37.8)$ & $2129(38.9)$ \\
\hline Caucasian, $n(\%)$ & $4549(86.2)$ & $5187(86.2)$ & $4141(82.3)$ & $4519(82.6)$ \\
\hline Non-Caucasian, $n(\%)$ & 730 (13.8) & $829(13.8)$ & $888(17.7)$ & $951(17.4)$ \\
\hline CHD, $n(\%)$ & $2129(40.3)$ & $2614(43.5)$ & $1181(23.5)$ & $1501(27.5)$ \\
\hline Diabetes mellitus, $n(\%)$ & $1491(28.2)$ & $1732(28.8)$ & $1591(31.6)$ & $1727(31.6)$ \\
\hline Metabolic syndrome, $n(\%)$ & $1912(46.5)$ & $2112(45.8)$ & $2321(54.7)$ & $2406(53.6)$ \\
\hline First-line, $n(\%)$ & $3008(57.0)$ & $3081(51.2)$ & $3249(64.6)$ & $3160(57.8)$ \\
\hline Second-line, $n(\%)$ & $2271(43.0)$ & $2935(48.8)$ & $1780(35.4)$ & $2310(42.2)$ \\
\hline $\mathrm{BMI} \geq 30 \mathrm{~kg} / \mathrm{m}^{2}, n(\%)$ & $1916(36.8)$ & $2206(37.2)$ & $2222(45.0)$ & $2331(43.3)$ \\
\hline BMI $\left(\mathrm{kg} / \mathrm{m}^{2}\right)$ & $(n=5201)$ & $(n=5931)$ & $(n=4942)$ & $(n=5384)$ \\
\hline Mean (SD) & $29.3(5.1)$ & $29.4(5.1)$ & $30.3(6.5)$ & $30.1(6.5)$ \\
\hline & & & & \\
\hline & $\begin{array}{l}\text { Statin } \\
(n=6682)\end{array}$ & $\begin{array}{l}\text { Statin/EZ } \\
(n=7250)\end{array}$ & $\begin{array}{l}\text { Statin } \\
(n=3626)\end{array}$ & $\begin{array}{l}\text { Statin/EZ } \\
(n=4236)\end{array}$ \\
\hline Mean age, years (SD) & $52.8(8.3)$ & $53.3(7.9)$ & $70.9(4.5)$ & $71.3(4.8)$ \\
\hline Male, $n(\%)$ & $3556(53.2)$ & 3909 (53.9) & $1723(47.5)$ & $2107(49.7)$ \\
\hline Female, $n(\%)$ & $3126(46.8)$ & $3341(46.1)$ & $1903(52.5)$ & $2129(50.3)$ \\
\hline Caucasian, $n(\%)$ & $5451(81.6)$ & $5962(82.2)$ & 3239 (89.3) & $3744(88.4)$ \\
\hline Non-Caucasian, $n(\%)$ & $1231(18.4)$ & $1288(17.8)$ & $387(10.7)$ & $492(11.6)$ \\
\hline CHD, $n(\%)$ & $1842(27.6)$ & $2189(30.2)$ & $1468(40.5)$ & $1926(45.5)$ \\
\hline Diabetes mellitus, $n(\%)$ & $1897(28.4)$ & $2077(28.6)$ & $1185(32.7)$ & $1382(32.6)$ \\
\hline Metabolic syndrome, $n(\%)$ & $2660(49.0)$ & $2734(47.3)$ & $1573(53.8)$ & $1784(53.8)$ \\
\hline First-line, $n(\%)$ & $4555(68.2)$ & $4465(61.6)$ & $1702(46.9)$ & $1776(41.9)$ \\
\hline Second-line, $n(\%)$ & $2127(31.8)$ & $2785(38.4)$ & $1924(53.1)$ & $2460(58.1)$ \\
\hline $\mathrm{BMI} \geq 30 \mathrm{~kg} / \mathrm{m}^{2}, n(\%)$ & $2910(44.4)$ & $3106(43.6)$ & $1228(34.2)$ & $1431(34.1)$ \\
\hline BMI $\left(\mathrm{kg} / \mathrm{m}^{2}\right)$ & $(n=6553)$ & $(n=7124)$ & $(n=3590)$ & $(n=4191)$ \\
\hline Mean (SD) & $30.3(6.2)$ & $30.2(6.1)$ & $28.9(5.1)$ & $28.9(5.1)$ \\
\hline
\end{tabular}


Table 2 (Continued)

\begin{tabular}{|c|c|c|c|c|c|c|}
\hline & \multicolumn{2}{|c|}{ White } & \multicolumn{2}{|c|}{ Black } & \multicolumn{2}{|c|}{ Other } \\
\hline & $\begin{array}{l}\text { Statin } \\
(n=8690)\end{array}$ & $\begin{array}{l}\text { Statin/EZ } \\
(n=9706)\end{array}$ & $\begin{array}{l}\text { Statin } \\
(n=722)\end{array}$ & $\begin{array}{l}\text { Statin/EZ } \\
(n=813)\end{array}$ & $\begin{array}{l}\text { Statin } \\
(n=896)\end{array}$ & $\begin{array}{l}\text { Statin/EZ } \\
(n=967)\end{array}$ \\
\hline Mean age, years (SD) & $59.7(11.2)$ & $60.3(11.0)$ & $56.0(11.1)$ & $57.7(11.1)$ & $56.4(11.3)$ & $57.3(11.4)$ \\
\hline$<65$ years, $n(\%)$ & $5451(62.7)$ & $5962(61.4)$ & $560(77.6)$ & $589(72.4)$ & $671(74.9)$ & $699(72.3)$ \\
\hline$\geq 65$ years, $n(\%)$ & 3239 (37.3) & $3744(38.6)$ & $162(22.4)$ & $224(27.6)$ & $225(25.1)$ & $268(27.7)$ \\
\hline Male, $n(\%)$ & $4549(52.3)$ & $5187(53.4)$ & $303(42.0)$ & $319(39.2)$ & $427(47.7)$ & $510(52.7)$ \\
\hline Female, $n(\%)$ & $4141(47.7)$ & 4519 (46.6) & $419(58.0)$ & $494(60.8)$ & $469(52.3)$ & $457(47.3)$ \\
\hline CHD, $n(\%)$ & $2894(33.3)$ & $3586(37.0)$ & $153(21.2)$ & $178(22.0)$ & $263(29.4)$ & $351(36.3)$ \\
\hline Diabetes mellitus, $n(\%)$ & $2407(27.7)$ & $2631(27.1)$ & $285(39.5)$ & $377(46.4)$ & $390(43.5)$ & $451(46.6)$ \\
\hline Metabolic syndrome, $n(\%)$ & $3532(49.8)$ & $3805(49.0)$ & $293(53.9)$ & $343(56.7)$ & $408(56.4)$ & $370(50.8)$ \\
\hline First-line, $n(\%)$ & $5138(59.1)$ & $5183(53.4)$ & $527(73.0)$ & $523(64.3)$ & $592(66.1)$ & $535(55.3)$ \\
\hline Second-line, $n(\%)$ & $3552(40.9)$ & $4523(46.6)$ & $195(27.0)$ & $290(35.7)$ & $304(33.9)$ & $432(44.7)$ \\
\hline $\mathrm{BMI} \geq 30 \mathrm{~kg} / \mathrm{m}^{2}, n(\%)$ & 3412 (39.9) & 3756 (39.3) & $407(57.2)$ & $482(60.0)$ & $319(36.0)$ & $299(31.3)$ \\
\hline BMI $\left(\mathrm{kg} / \mathrm{m}^{2}\right)$ & $(n=8546)$ & $(n=9557)$ & $(n=712)$ & $(n=804)$ & $(n=885)$ & $(n=954)$ \\
\hline \multirow[t]{3}{*}{ Mean (SD) } & $29.7(5.8)$ & $29.6(5.7)$ & $32.2(6.9)$ & $32.4(6.3)$ & $29.0(5.5)$ & $28.4(5.5)$ \\
\hline & \multicolumn{3}{|c|}{ First-line } & \multicolumn{3}{|c|}{ Second-line } \\
\hline & \multicolumn{2}{|c|}{$\begin{array}{l}\text { Statin } \\
(n=6257)\end{array}$} & $\begin{array}{l}\text { Statin/EZ } \\
(n=6241)\end{array}$ & \multicolumn{2}{|c|}{$\begin{array}{l}\text { Statin } \\
(n=4051)\end{array}$} & $\begin{array}{l}\text { Statin/EZ } \\
(n=5245)\end{array}$ \\
\hline Mean age, years (SD) & \multicolumn{2}{|c|}{$57.3(10.9)$} & $57.9(10.7)$ & \multicolumn{2}{|c|}{$62.1(11.2)$} & $62.2(11.1)$ \\
\hline$<65$ years, $n(\%)$ & \multicolumn{2}{|c|}{$4555(72.8)$} & 4465 (71.5) & \multicolumn{2}{|c|}{$2127(52.5)$} & $2785(53.1)$ \\
\hline$\geq 65$ years, $n(\%)$ & \multicolumn{2}{|c|}{$1702(27.2)$} & $1776(28.5)$ & \multicolumn{2}{|c|}{$1924(47.5)$} & $2460(46.9)$ \\
\hline Male, $n(\%)$ & \multicolumn{2}{|c|}{$3008(48.1)$} & 3081 (49.4) & \multicolumn{2}{|c|}{$2271(56.1)$} & $2935(56.0)$ \\
\hline Female, $n(\%)$ & \multicolumn{2}{|c|}{3249 (51.9) } & $3160(50.6)$ & \multicolumn{2}{|c|}{$1780(43.9)$} & $2310(44.0)$ \\
\hline Caucasian, $n(\%)$ & \multicolumn{2}{|c|}{$5138(82.1)$} & $5183(83.0)$ & \multicolumn{2}{|c|}{$3552(87.7)$} & $4523(86.2)$ \\
\hline Non-Caucasian, $n(\%)$ & \multicolumn{2}{|c|}{$1119(17.9)$} & $1058(17.0)$ & \multicolumn{2}{|c|}{$499(12.3)$} & $722(13.8)$ \\
\hline CHD, $n(\%)$ & \multicolumn{2}{|c|}{$1019(16.3)$} & $1194(19.1)$ & \multicolumn{2}{|c|}{$2291(56.6)$} & $2921(55.8)$ \\
\hline Diabetes mellitus, $n(\%)$ & \multicolumn{2}{|c|}{$1751(28.0)$} & $1569(25.1)$ & 1331 & 2.9) & $1890(36.0)$ \\
\hline Metabolic syndrome, $n(\%)$ & 1364 & 3.8) & $1938(55.5)$ & 2869 & 9.3) & $2580(46.0)$ \\
\hline $\mathrm{BMI} \geq 30 \mathrm{~kg} / \mathrm{m}^{2}, n(\%)$ & 2685 & 3.9) & $2538(41.6)$ & 1453 & $6.0)$ & 1999 (38.3) \\
\hline BMI $\left(\mathrm{kg} / \mathrm{m}^{2}\right)$ & $(n=$ & 14) & $(n=6103)$ & $(n=$ & 29) & $(n=5212)$ \\
\hline Mean (SD) & 30.2 & & $29.9(5.9)$ & 29.2 & & $29.5(5.7)$ \\
\hline
\end{tabular}

BMI, body mass index; CHD, coronary heart disease; EZ, ezetimibe; SD, standard deviation.

and allergic reaction or rash-related (see specific terms in footnote of Table 3 ). Safety populations were defined based on their definitions at the time of each individual study.

Laboratory AEs were analyzed in patients who had $\geq 1$ post-baseline assessment; and special AEs of interest were analyzed in All Patients as Treated (APaT). In the full cohort, the percentage of patients with AEs within treatment groups was assessed by a logistic regression model with terms for first-line/second-line therapy (statin-naïve or rendered statinnaïve by washout/ongoing statin at study entry), trial within first-/second-line therapy, and treatment. The same model was fitted for age $(<65, \geq 65$ years), sex (male, female), race (white, black, other) and firstline/second-line subgroups with additional terms for subgroup and subgroup-by-treatment interaction. For rare AEs (those with a rate $<1 \%$ for both statin and statin plus ezetimibe treatments), the data were analyzed by a Poisson regression model. Due to the paucity of data, the trial within first-/second-line term coefficient could not be estimated in the Poisson regression model and was therefore excluded. For the full cohort, a sensitivity analysis was performed by way of risk difference using a logistic mixed model and Miettinen \& Nurminen method with 'effective sample size'. The logistic mixed model included terms for first-/second-line study, treatment, and random effect for study.

\section{Results}

There were 10,542 patients randomized to the statin monotherapy groups and 11,746 subjects randomized 
Table 3 Summary of safety data in the full cohort

\begin{tabular}{|c|c|c|c|}
\hline & Statin & Statin/EZ & $p$-value for treatment \\
\hline AE Summary, $n(\%)$ & $(N=10,517)$ & $(N=11,714)$ & \\
\hline$\geq 1 \mathrm{AE}$ & 3455 (32.85) & 3717 (31.73) & 0.547 \\
\hline Drug-related* AE & $833(7.92)$ & $961(8.20)$ & 0.163 \\
\hline Serious AEs (SAEs) & $145(1.38)$ & $187(1.60)$ & 0.202 \\
\hline Drug-related* SAEs & $6(0.06)$ & $13(0.11)$ & 0.148 \\
\hline Death & $5(0.05)$ & $7(0.06)$ & 0.700 \\
\hline \multicolumn{4}{|l|}{ Discontinuations } \\
\hline Due to an $A E$ & $219(2.08)$ & $263(2.25)$ & 0.337 \\
\hline Due to drug-related* $A E$ & $136(1.29)$ & $177(1.51)$ & 0.133 \\
\hline Due to SAEs & $34(0.32)$ & $38(0.32)$ & 0.899 \\
\hline Due to drug-related ${ }^{*}$ SAEs & $6(0.06)$ & $7(0.06)$ & 0.866 \\
\hline \multicolumn{4}{|l|}{ Laboratory Values, $\mathrm{m} / \boldsymbol{n}(\%)$} \\
\hline ALT $\geq 3 \times$ ULN, consecutive & $31 / 10,341(0.30)$ & $50 / 11,512(0.43)$ & 0.084 \\
\hline AST $\geq 3 \times$ ULN, consecutive & $23 / 10,342(0.22)$ & $30 / 11,512(0.26)$ & 0.525 \\
\hline ALT or AST $\geq 3 \times$ ULN, consecutive & $36 / 10,342(0.35)$ & $64 / 11,512(0.56)$ & 0.017 \\
\hline $\mathrm{CK} \geq 10 \times \mathrm{ULN}$ & $13 / 10,342(0.13)$ & $9 / 11,514(0.08)$ & 0.350 \\
\hline Myopathy $\dagger$ & $4 / 10,342(0.04)$ & $3 / 11,512(0.03)$ & 0.67 \\
\hline Rhabdomyolysisł & $0 / 10,342(0.00)$ & $0 / 11,512(0.00)$ & $\mathrm{N} / \mathrm{A}$ \\
\hline Specific AE groups, $\boldsymbol{n}(\%)$ & $(n=10,505)$ & $(n=11,705)$ & \\
\hline Hepatitis-related§ & $8(0.08)$ & $5(0.04)$ & 0.359 \\
\hline Gallbladder-related & $10(0.10)$ & $14(0.12)$ & 0.522 \\
\hline Gastrointestinal-related ${ }^{* *}$ & $792(7.54)$ & $832(7.11)$ & 0.112 \\
\hline Allergic reaction or rash $\uparrow \dagger$ & $139(1.32)$ & $185(1.58)$ & 0.254 \\
\hline
\end{tabular}

Definitions of specific AE groups apply to all subgroup tables:

${ }^{*}$ Considered by the investigator to be possibly, probably or definitely related to study drug.

${ }^{\dagger}$ Myopathy is defined as CK elevation > $10 \times$ ULN with associated muscle symptoms with no other explanatory cause.

${ }^{\ddagger}$ Rhabdomyolysis is defined as myopathy with associated evidence of renal damage.

§In addition to review of the effects of ezetimibe + statin on laboratory parameters associated with liver function, "hepatitis-related" clinical AE terms (preferred MedDRA terms) were pre-identified for collective review, and included cholestasis, hepatitis, hepatic lesion, hepatomegaly, hepatic cyst, hepatitis cholestatic.

"For gallbladder-related clinical AEs the preferred terms (preferred MedDRA terms) were pre-identified for collective review, including bile duct obstruction, biliary colic, cholangitis, cholecystitis, cholecystitis chronic, cholelithiasis, and gallbladder disorder.

${ }^{* *}$ For gastrointestinal-related clinical AEs, the preferred terms (preferred MedDRA terms) were pre-identified for collective review, and included abdominal discomfort, abdominal distension, abdominal pain, abdominal tenderness, colitis, colonic polyp, constipation, dental caries, dental discomfort, diarrhoea, diverticulum, duodenitis, dyspepsiea, dysphagia, erosive duodenitis, faeces discolored, flatulence, food poisoning, gastritis, gastroesophageal reflux disease, gingival pain, haemorrhoids, hiatus hernia, nausea, oesophageal stenosis, rectal haemorrhage, tooth loss, toothache, and vomiting.

${ }^{\dagger}$ For allergic reaction or rash the preferred terms (preferred MedDRA terms) were pre-identified for collective review, and included analphylaxis, angioedema, dermatitis, dermagraphism, drug hypersensitivity, eczema, eosinophilia, erythema, face oedema, hypersensitivity, palmar erythema, periorbital oedema, photodermatosis, photosensitivity, pigmentation disorder, priuritus, rash, rosacea, skin disorder, skin exfoliation, skin hyperpigmentation, skin inflammation, skin lesion, subcutaneious nodule, systemic lupus erythematosus rash, and urticaria.

to the statin + ezetimibe combination groups within the studies included. A total of 22,278 patients received at least one dose of study drug and were included in the safety analysis (Table 1).

\section{Baseline characteristics}

Baseline characteristics were generally similar between treatment groups in the full cohort (Table 2). The majority of the intent-to-treat (ITT) population was Caucasian and the mean age ( \pm standard deviation) was $60 \pm 11$ years with a mean body mass index (BMI) of $29.8 \mathrm{~kg} / \mathrm{m}^{2}$. Half of subjects $(50.1 \%$ ) had metabolic syndrome, and approximately $1 / 3$ of subjects had CHD (34\%) or diabetes mellitus (30\%). At baseline, $57 \%$ of subjects were treated as first-line, and these subjects received statin monotherapy more often than receiving combination statin/ezetimibe (61\% vs. $54 \%)$.

In the subgroups that were compared (Table 2), a smaller proportion of women had CHD compared 
with men, and a greater proportion of women had metabolic syndrome compared with men. Compared with older subjects ( $\geq 65$ years), younger subjects ( $<65$ years) had a higher proportion of non-Caucasians and a higher proportion of subjects that were treated with first-line therapy. The older group included a higher proportion of subjects with CHD, diabetes mellitus, and metabolic syndrome compared with the younger age group. Compared with the White and Other subgroups, Black subjects had a higher proportion of females and subjects treated with first-line therapy. Blacks had the lowest proportion of subjects with CHD, while Whites had the lowest proportion of subjects with diabetes mellitus and metabolic syndrome. When comparing first-line with second-line studies, subjects that were treated with second-line therapy tended to be older by approximately 4.5 years, and included a higher proportion of males, subjects with CHD, and subjects with diabetes mellitus, but included a smaller proportion of subjects with metabolic syndrome and non-Caucasians. Although mean BMI was similar between all subgroups, women, subjects $\geq 65$ years, Black subjects and subjects treated first-line had higher proportions with BMI $\geq 30 \mathrm{~kg} / \mathrm{m}^{2}$.

\section{Safety results}

In the full cohort (Table 3), the only significant difference between treatments was in consecutive AST or ALT elevations $\geq 3 \times$ ULN. Although the incidence $(0.35 \%$ vs. $0.56 \%$, statin vs. statin/ezetimibe) was small in both treatment groups, there were significantly more reports of elevations in subjects treated with ezetimibe/statin therapy $(\mathrm{p}=0.017)$. Otherwise, both treatments had generally similar tolerability and safety profiles (i.e., there were no between-treatment differences in the proportion of subjects reporting $\geq 1 \mathrm{AEs}$, drug-related AEs, serious AEs, serious drug-related AEs, discontinuations due to AEs, or CK elevations $\geq 10 \times \mathrm{ULN}$ ). There was no difference in the incidence of myopathy, which was small in both treatment groups $(0.03 \%-0.04 \%)$, and there were no cases of rhabdomyolysis in any of the studies. A total of 12 deaths were reported during

Table 4 Summary of safety data in men and women

\begin{tabular}{|c|c|c|c|c|c|c|c|}
\hline & \multicolumn{2}{|l|}{ Male } & \multicolumn{2}{|l|}{ Female } & \multicolumn{3}{|c|}{$p$-value for effects } \\
\hline & Statin & Statin/EZ & Statin & Statin/EZ & Treatment & Sex & $\begin{array}{l}\text { Sex by treatment } \\
\text { interaction }\end{array}$ \\
\hline AE summary, $n(\%)$ & $(n=5380)$ & $(n=6129)$ & $(n=5137)$ & $(n=5585)$ & & & \\
\hline$\geq 1 \mathrm{AE}$ & $1537(28.57)$ & $1779(29.03)$ & $1918(37.34)$ & $1938(34.70)$ & - & $<0.001$ & 0.014 \\
\hline Drug-related* $\mathrm{AE}$ & $349(6.49)$ & $456(7.44)$ & $484(9.42)$ & $505(9.04)$ & 0.017 & $<0.001$ & - \\
\hline Serious AEs (SAEs) & $74(1.38)$ & $107(1.75)$ & $71(1.38)$ & $80(1.43)$ & - & - & - \\
\hline Drug-related* SAEs & $2(0.04)$ & $8(0.13)$ & $4(0.08)$ & $5(0.09)$ & - & - & - \\
\hline Death & $4(0.07)$ & $3(0.05)$ & $1(0.02)$ & $4(0.07)$ & - & - & - \\
\hline \multicolumn{8}{|l|}{ Discontinuations } \\
\hline Due to an $A E$ & $91(1.69)$ & $105(1.71)$ & $128(2.49)$ & $158(2.83)$ & - & 0.016 & - \\
\hline Due to drug-related* $\mathrm{AE}$ & $49(0.91)$ & $69(1.13)$ & $87(1.69)$ & $108(1.93)$ & - & 0.002 & - \\
\hline Due to SAEs & $18(0.33)$ & $23(0.38)$ & $16(0.31)$ & $15(0.27)$ & - & - & - \\
\hline Due to drug-related* SAEs & $2(0.04)$ & $4(0.07)$ & $4(0.08)$ & $3(0.05)$ & - & - & - \\
\hline \multicolumn{8}{|l|}{ Laboratory values, $\mathrm{m} / \boldsymbol{n}(\%)$} \\
\hline ALT $\geq 3 \times$ ULN, consecutive & $15 / 5289(0.28)$ & $34 / 6031(0.56)$ & $16 / 5052(0.32)$ & $16 / 5481(0.29)$ & 0.018 & - & - \\
\hline AST $\geq 3 \times$ ULN, consecutive & $7 / 5290(0.13)$ & $16 / 6031(0.27)$ & $16 / 5052(0.32)$ & $14 / 5481(0.26)$ & - & 0.049 & - \\
\hline $\begin{array}{l}\text { ALT or AST } \geq 3 \times U L N, \\
\text { consecutive }\end{array}$ & $17 / 5290(0.32)$ & $41 / 6031(0.68)$ & $19 / 5052(0.38)$ & $23 / 5481(0.42)$ & 0.005 & - & - \\
\hline $\mathrm{CK} \geq 10 \times \mathrm{ULN}$ & $9 / 5290(0.17)$ & $7 / 6033(0.12)$ & $4 / 5052(0.08)$ & $2 / 5481(0.04)$ & - & - & - \\
\hline Myopathy & $2 / 5290(0.04)$ & $2 / 6033(0.03)$ & $2 / 5052(0.04)$ & $1 / 5481(0.02)$ & - & - & - \\
\hline Rhabdomyolysis & $0 / 5290(0.00)$ & $0 / 6033(0.00)$ & $0 / 5052(0.00)$ & $0 / 5481(0.00)$ & - & - & - \\
\hline Specific AE groups ${ }^{\dagger}, n(\%)$ & $(n=5372)$ & $(n=6124)$ & $(n=5133)$ & $(n=5581)$ & & & \\
\hline Hepatitis-related & $4(0.07)$ & $4(0.07)$ & $4(0.08)$ & $1(0.02)$ & - & - & - \\
\hline Gallbladder-related & $3(0.06)$ & $6(0.10)$ & $7(0.14)$ & $8(0.14)$ & - & - & - \\
\hline Gastrointestinal-related & $324(6.03)$ & $370(6.04)$ & $468(9.12)$ & $462(8.28)$ & - & $<.001$ & - \\
\hline Allergic reaction or rash & $50(0.93)$ & $79(1.29)$ & $89(1.73)$ & $106(1.90)$ & - & $<.001$ & - \\
\hline
\end{tabular}

*Considered by the investigator to be possibly, probably or definitely related to study drug.

${ }^{\dagger}$ Definitions of specific AE groups are the same as those in Table 3. 
the course of all 27 studies and none of the deaths were attributed to treatment. The causes of the five deaths in the statin group were due to head injury after a fall, chronic obstructive pulmonary disease, sudden cardiac death, mixed drug ingestion, and myocardial infarction. The causes of the seven deaths in the statin/ezetimibe group were suicide, two deaths due to head injury, hypotension/respiratory failure, cardiac arrest, stroke, and spontaneous abortion leading to fetal death. The results of the sensitivity analysis were consistent with the original logistic regression model analysis, with statistically significantly greater reports in consecutive AST or ALT elevations $\geq 3 \times U L N$ in ezetimibe/statin-treated subjects compared with statin monotherapy-treated subjects $(\mathrm{p}=0.041)$.

Generally, higher AE rates were reported for women regardless of treatment (Table 4). When comparing the sexes, women reported significantly more AEs (i.e., $\geq 1 \mathrm{AE}$ ), drug-related AEs, discontinuations due to AEs, discontinuations due to drug-related AEs, gastrointestinal-related AEs, allergic reaction- or rash- related AEs and AST elevations $\geq 3 \times \mathrm{ULN}$ than men. Among men, a significantly greater proportion treated with the combination of statin/ezetimibe reported AST or ALT elevations $\geq 3 \times U L N$, and ALT elevations $\geq 3 \times$ ULN than those treated with statin alone. There were no significant differences between men and women in the occurrence of myopathy, which was small in both sexes.

Generally similar rates of AEs were reported between the age groups (Table 5), although significantly more reports of drug-related serious AEs occurred in the statin/ezetimibe group and this was driven mainly by reports in subjects $<65$ years. Also among subjects $<65$ years of age, those being treated with the combination statin/ezetimibe reported significantly more discontinuations due to drug-related serious AEs, and ALT and/or AST elevations $\geq 3 \times$ ULN compared with subjects $\geq 65$ years treated with statin alone. Significantly more subjects $\geq 65$ years reported serious AEs and discontinued due to serious AEs compared with subjects $<65$ years, whereas significantly more subjects

Table 5 Summary of safety data by age group

\begin{tabular}{|c|c|c|c|c|c|c|c|}
\hline & Statin & Statin/EZ & Statin & Statin/EZ & Treatment & Age & $\begin{array}{l}\text { Age by } \\
\text { treatment } \\
\text { interaction }\end{array}$ \\
\hline AE summary, $n(\%)$ & $(n=6816)$ & $(n=7406)$ & $(n=3701)$ & $(n=4308)$ & & & \\
\hline$\geq 1 \mathrm{AE}$ & $2368(34.74)$ & $2426(32.76)$ & $1087(29.37)$ & $1291(29.97)$ & - & - & - \\
\hline Serious AEs (SAEs) & $74(1.09)$ & $93(1.26)$ & $71(1.92)$ & $94(2.18)$ & - & $<0.001$ & - \\
\hline Drug-related* SAEs & $3(0.04)$ & $13(0.18)$ & $3(0.08)$ & $0(0.00)$ & 0.014 & - & 0.003 \\
\hline Death & $4(0.06)$ & $3(0.04)$ & $1(0.03)$ & $4(0.09)$ & - & - & - \\
\hline \multicolumn{8}{|l|}{ Discontinuations } \\
\hline Due to an $A E$ & $137(2.01)$ & $160(2.16)$ & $82(2.22)$ & $103(2.39)$ & - & - & - \\
\hline Due to drug-related* $A E$ & $90(1.32)$ & $114(1.54)$ & $46(1.24)$ & $63(1.46)$ & - & - & - \\
\hline AST $\geq 3 \times$ ULN, consecutive & $12 / 6699(0.18)$ & $24 / 7270(0.33)$ & $11 / 3643(0.30)$ & $6 / 4242(0.14)$ & - & - & 0.023 \\
\hline ALT or AST $\geq 3 \times$ ULN, consecutive & $23 / 6699(0.34)$ & $56 / 7270(0.77)$ & $13 / 3643(0.36)$ & $8 / 4242(0.19)$ & 0.001 & - & 0.004 \\
\hline $\mathrm{CK} \geq 10 \times \mathrm{ULN}$ & $12 / 6699(0.18)$ & $7 / 7272(0.10)$ & $1 / 3643(0.03)$ & $2 / 4242(0.05)$ & - & 0.049 & - \\
\hline Myopathy & $3 / 6699(0.03)$ & $2 / 7272(0.02)$ & $1 / 3643(0.03)$ & $1 / 4242(0.02)$ & - & - & - \\
\hline Rhabdomyolysis & $0 / 6699(0.00)$ & $0 / 7272(0.00)$ & $0 / 3643(0.00)$ & $0 / 4242(0.00)$ & - & - & - \\
\hline Specific AE groups ${ }^{\dagger}, n(\%)$ & $(n=6809)$ & $(n=7401)$ & $(n=3696)$ & $(n=4304)$ & & & \\
\hline Hepatitis-related & $6(0.09)$ & $3(0.04)$ & $2(0.05)$ & $2(0.05)$ & - & - & - \\
\hline Gallbladder-related & $7(0.10)$ & $5(0.07)$ & $3(0.08)$ & $9(0.21)$ & - & - & - \\
\hline Gastrointestinal-related & $543(7.97)$ & $542(7.32)$ & $249(6.74)$ & $290(6.74)$ & - & - & - \\
\hline Allergic reaction or rash & $102(1.50)$ & $124(1.68)$ & $37(1.00)$ & $61(1.42)$ & - & - & - \\
\hline
\end{tabular}


$<65$ years reported CK elevations $\geq 10 \times$ ULN compared with subjects $\geq 65$ years. There were no significant differences between subjects $<65$ years and $\geq 65$ years in the occurrence of myopathy regardless of treatment, which was small in both age groups.

Likewise, there were generally similar rates of AEs reported between race subgroups (Table 6), with a few exceptions. Subjects in the 'Other' subgroup reported significantly fewer AEs (i.e., $\geq 1 \mathrm{AE}$ ) and drug-related AEs compared with Black or White subjects. Among White subjects, significantly more who were treated with the combinations of statin/ezetimibe experienced AST or ALT elevations $\geq 3 \times \mathrm{ULN}$ compared with White subjects treated with statin monotherapy, although the incidence was low. There was no other difference between treatments in the Black or Other subgroups. Finally, although the proportions were very small $(<1 \%)$ in both treatment groups in all three race subgroups overall, among Black subjects, there was a significantly higher proportion of CK elevations $\geq 10 \times \mathrm{ULN}$ treated with statin monotherapy compared with statin/ezetimibe combination $(0.69 \%$ vs. $0.0 \%)$. There were no significant differences between race groups in the occurrence of myopathy, which was low in all three groups.

There was a significant effect of treatment, as well as a first-line/second-line by treatment interaction on serious AEs, with more reports in the combination of statin/ezetimibe vs. statin monotherapy in subjects who were taking ongoing statin at study entry (subjects treated second-line) (Table 7). There was also a significant effect of treatment on allergic reaction or rash AEs, with more reports in the combination of statin/ezetimibe vs. statin monotherapy in both first-line and subjects treated second-line (Table 7). A significantly greater proportion of subjects who were treated first-line reported $\geq 1 \mathrm{AE}$, drug-related AEs, serious AEs, potentially hepatitisrelated AEs, gastrointestinal-related AEs, allergic

Table 6 Summary of safety data by race groups

\begin{tabular}{|c|c|c|c|c|c|c|c|c|c|}
\hline & Statin & Statin/EZ & Statin & Statin/EZ & Statin & Statin/EZ & Treatment & Race & $\begin{array}{l}\text { Race by } \\
\text { treatment } \\
\text { interaction }\end{array}$ \\
\hline AE summary, $n(\%)$ & $(n=8837)$ & $(n=9888)$ & $(n=738)$ & $(n=830)$ & $(n=942)$ & $(n=996)$ & & & \\
\hline$\geq 1 \mathrm{AE}$ & $2911(32.94)$ & $3170(32.06)$ & $256(34.69)$ & $279(33.61)$ & $288(30.57)$ & $268(26.91)$ & - & 0.048 & - \\
\hline Serious AEs (SAEs) & $127(1.44)$ & $165(1.67)$ & $10(1.36)$ & $12(1.45)$ & $8(0.85)$ & $10(1.00)$ & - & - & - \\
\hline Drug-related* SAEs & $5(0.06)$ & $9(0.09)$ & $1(0.14)$ & $1(0.12)$ & $0(0.00)$ & $3(0.30)$ & - & - & - \\
\hline Death & $5(0.06)$ & $6(0.06)$ & $0(0.00)$ & $0(0.00)$ & $0(0.00)$ & $1(0.10)$ & - & - & - \\
\hline Discontinuations & & & & & & & & - & - \\
\hline Due to an $A E$ & $188(2.13)$ & $232(2.35)$ & $16(2.17)$ & $13(1.57)$ & $15(1.59)$ & $18(1.81)$ & - & - & - \\
\hline Due to drug-related* $A E$ & $118(1.34)$ & $158(1.60)$ & $9(1.22)$ & $7(0.84)$ & $9(0.96)$ & $12(1.20)$ & - & - & - \\
\hline AST $\geq 3 \times$ ULN, consecutive & $20 / 8702(0.23)$ & $24 / 9726(0.25)$ & $0 / 727(0.00)$ & $0 / 810(0.00)$ & $3 / 913(0.33)$ & $6 / 976(0.61)$ & - & 0.004 & - \\
\hline $\begin{array}{l}\text { ALT or AST } \geq 3 \times \text { ULN, } \\
\text { consecutive }\end{array}$ & 29/8702 (0.33) & $53 / 9726(0.54)$ & $2 / 727(0.28)$ & $3 / 810(0.37)$ & $5 / 913(0.55)$ & $8 / 976(0.82)$ & 0.024 & - & - \\
\hline$C K \geq 10 \times$ ULN & $7 / 8702(0.08)$ & $6 / 9728(0.06)$ & $5 / 727(0.69)$ & $0 / 810(0.00)$ & $1 / 913(0.11)$ & $3 / 976(0.31)$ & - & 0.008 & 0.023 \\
\hline Myopathy & $2 / 8702(0.02)$ & $1 / 9728(0.01)$ & $1 / 727(0.14)$ & $1 / 810(0.12)$ & $1 / 913(0.11)$ & $1 / 976(0.10)$ & - & - & - \\
\hline Rhabdomyolysis & $0 / 8702(0.00)$ & $0 / 9728(0.00)$ & $0 / 727(0.00)$ & $0 / 810(0.00)$ & $0 / 913(0.00)$ & $3 / 976(0.31)$ & - & - & - \\
\hline Specific AE groups ${ }^{\dagger}, n(\%)$ & $(n=8842)$ & $(n=9893)$ & $(n=737)$ & $(n=828)$ & $(n=926)$ & $(n=983)$ & & & \\
\hline Hepatitis-related & $8(0.09)$ & $4(0.04)$ & $0(0.00)$ & $0(0.00)$ & $0(0.00)$ & $1(0.10)$ & - & - & - \\
\hline Gallbladder-related & $10(0.11)$ & $13(0.13)$ & $0(0.00)$ & $0(0.00)$ & $0(0.00)$ & $1(0.10)$ & - & - & - \\
\hline Gastrointestinal-related & $675(7.63)$ & $720(7.28)$ & $48(6.51)$ & $59(7.13)$ & $69(7.45)$ & $53(5.39)$ & - & - & - \\
\hline Allergic reaction or rash & $114(1.29)$ & $160(1.62)$ & $8(1.09)$ & $14(1.69)$ & $17(1.84)$ & $11(1.12)$ & - & - & - \\
\hline
\end{tabular}


Table 7. Summary of safety data in patients grouped by those receiving first-line treatment or second-line treatment

\begin{tabular}{|c|c|c|c|c|c|c|c|}
\hline & Statin & Statin/EZ & Statin & Statin/EZ & Treatment & $\begin{array}{l}\text { First/second } \\
\text { line }\end{array}$ & $\begin{array}{l}\text { First-/second-line } \\
\text { by treatment } \\
\text { interaction }\end{array}$ \\
\hline AE summary, $n(\%)$ & $(n=6694)$ & $(n=6664)$ & $(n=3823)$ & $(n=5050)$ & & & \\
\hline$\geq 1 \mathrm{AE}$ & $2553(38.14)$ & $2510(37.67)$ & $902(23.59)$ & $1207(29.90)$ & - & $<0.001$ & - \\
\hline Serious AEs (SAEs) & $95(1.42)$ & $91(1.37)$ & $50(1.31)$ & $96(1.90)$ & 0.016 & - & 0.035 \\
\hline Drug-related* SAEs & $4(0.06)$ & $9(0.14)$ & $2(0.05)$ & $4(0.08)$ & - & - & - \\
\hline Death & $4(0.06)$ & $3(0.04)$ & $2(0.05)$ & $3(0.06)$ & - & - & - \\
\hline \multicolumn{8}{|l|}{ Discontinuations } \\
\hline Due to an $A E$ & $150(2.24)$ & $170(2.55)$ & $69(1.80)$ & $93(1.84)$ & - & - & - \\
\hline Due to drug-related* $\mathrm{AE}$ & $91(1.36)$ & $116(1.74)$ & $45(1.18)$ & $61(1.21)$ & - & - & - \\
\hline AST $\geq 3 \times$ ULN, consecutive & $18 / 6587(0.27)$ & $17 / 6557(0.26)$ & $5 / 3755(0.13)$ & $13 / 4955(0.26)$ & - & - & - \\
\hline $\begin{array}{l}\text { ALT or AST } \geq 3 \times \text { ULN, } \\
\text { consecutive }\end{array}$ & $26 / 6587(0.39)$ & $41 / 6557(0.63)$ & $10 / 3755(0.27)$ & $23 / 4955(0.46)$ & - & - & - \\
\hline CK $\geq 10 \times$ ULN & $12 / 6587(0.18)$ & $7 / 6558(0.11)$ & $1 / 3755(0.03)$ & $2 / 4956(0.04)$ & - & 0.016 & - \\
\hline Myopathy & $3 / 6587(0.05)$ & $3 / 6558(0.05)$ & $1 / 3755(0.03)$ & $0 / 4956(0.00)$ & - & - & - \\
\hline Rhabdomyolysis & $0 / 6587(0.00)$ & $0 / 6558(0.00)$ & $0 / 3755(0.00)$ & $\mathrm{N} / \mathrm{A}$ & - & - & - \\
\hline Specific AE groups ${ }^{\dagger}, \boldsymbol{n}(\%)$ & $(n=6684)$ & $(n=6662)$ & $(n=3821)$ & $(n=5043)$ & & & \\
\hline Hepatitis-related & $8(0.12)$ & $5(0.08)$ & $0(0.00)$ & $0(0.00)$ & - & $<0.001$ & - \\
\hline Gallbladder-related & $8(0.12)$ & $7(0.11)$ & $2(0.05)$ & $7(0.14)$ & - & - & - \\
\hline Gastrointestinal-related & $618(9.25)$ & $572(8.59)$ & $174(4.55)$ & $260(5.16)$ & - & $<0.001$ & 0.010 \\
\hline Allergic reaction or rash & $122(1.83)$ & $140(2.10)$ & $17(0.44)$ & $45(0.89)$ & 0.030 & $<0.001$ & 0.044 \\
\hline
\end{tabular}

reaction or rash $\mathrm{AEs}$, and $\mathrm{CK}$ elevations $\geq 10 \times \mathrm{ULN}$ compared with subjects treated second-line in both treatment groups (Table 7). There was no significant difference between subjects treated first-line compared with second-line in the occurrence of myopathy, which was low overall.

\section{Discussion}

The efficacy and safety profiles of ezetimibe, statins, and the combination of ezetimibe with various doses of statins have been well-established by numerous clinical trials in the general population. The accumulation of study data into a large database from 27 studies conducted over several years provided the opportunity to assess the safety and tolerability profiles of ezetimibe combined with statins in the overall population and in population subgroups with increased statistical power. The results of this pooled analysis confirm that the overall safety and tolerability profiles of ezetimibe added to a statin are generally comparable to statin monotherapy for up to 24 weeks. Irrespective of treatment group, generally higher rates of AEs were reported in women compared with men, and generally similar rates of AEs were reported between races and between age groups. More AEs were reported by subjects in first-line studies than subjects in second-line studies. The occurrence of myopathy was low and did not differ between treatments or any of the subgroups studied.

Although the incidence was low, elevations in liver enzymes were observed more often with ezetimibe/statin combination treatment compared with statin monotherapy in the overall population and in most subgroups studied. These rates were consistent with the incidence reported in the prescribing information for statin monotherapy $(0.7 \%-1.9 \%)$ and ezetimibe combined with simvastatin (Vytorin; $1.7 \%)(13,14)$ Similarly, in an efficacy and safety analysis conducted in the same database as this 
pooled analysis, but in both subgroups of patients with and without diabetes, consecutive or presumed consecutive elevations $>3 \times$ ULN in liver enzymes were also noted somewhat more frequently in subjects receiving ezetimibe/statin combination compared with statin monotherapy (12).

There was a statistically significant treatment effect reported for CK elevations $\geq 10 \times$ ULN among Black subjects, with increases observed significantly more often with statin monotherapy vs. the combination treatment. However, these elevations resulted in myopathy in only two cases, one in each treatment group. Given the small number of events, this was likely a chance imbalance. No such racial imbalances in the incidence of myopathy have been identified in the literature. Additional safety studies with an even greater number of Black subjects and prespecified endpoints are needed to produce conclusive evidence about muscle-related safety with statin and/or ezetimibe-plus-statin treatment.

Interestingly, although there was no treatment effect among subjects grouped by age, there was an age effect with more subjects in the younger age group reporting $\mathrm{CK}$ elevations $\geq 10 \times \mathrm{ULN}$ compared with older subjects. Based on what is known about the increased risk of myopathy, (which involves CK elevations) in older subjects, one would predict that more subjects in the older age group would have CK elevations $\geq 10 \times$ ULN compared with younger subjects $(5,15)$. One explanation may be that there was a much higher proportion of subjects $<65$ years included in the first-line studies compared with second-line studies, which also reported a significantly greater effect on CK elevations $\geq 10 \times \mathrm{ULN}$, and therefore many older subjects included in this pooled analysis may have been screened out for adverse effects or risk for CK elevations. Indeed, screening out for tolerability issues may explain why subjects treated second-line reported significantly fewer clinical and laboratory AEs compared with subjects treated first-line. In the second-line studies, subjects who experienced AEs most likely did not continue to randomization.

There were some differences between the sexes, with women reporting significantly more clinical AEs and discontinued treatment due to AEs compared with men, regardless of treatment. In addition to potential tolerability issues in women, liver enzyme elevations were more common with the combination treatment than with statin monotherapy in men, but not in women. More than $40 \%$ of the men included in these trials were diagnosed with $\mathrm{CHD}$ at baseline compared with $26 \%$ among women. It is possible that men were taking more concomitant medications, which affected drug metabolism and contributed to elevations in liver enzymes. An evaluation of ALT and CK elevation in an ambulatory care setting $(N=4958)$ found that significant elevations in either ALT or CK during ezetimibe treatment is usually associated with concomitant medications and that rates of ALT and/or CK elevations were similar to that of placebo (16).

\section{Limitations}

These trials were short-term (6-24 weeks, mean duration of follow-up $=9$ weeks), and therefore could not assess long-term safety and tolerability. However, long-term trials have also documented the safety of ezetimibe combined with statin therapy (17-20). In addition, many patients had been receiving statins prior to enrolling in the trials, and this likely would influence the on-study AE profile. Further studies assessing tolerability in patients without previous statin treatment may help clarify whether specific subgroups are more likely to experience particular AEs. Although rosuvastatin was assessed as a monotherapy, there were no treatment arms that included the combination of ezetimibe with rosuvastatin included in this database. However, clinical experience suggests that the safety and tolerability profiles of that combination would be generally similar to other statin/ezetimibe combinations (21-23).

\section{Conclusion}

In conclusion, ezetimibe added to a statin and statin monotherapy both provide generally safe and welltolerated therapeutic options for dyslipidemia. These results describe the safety profiles over 6-24 weeks of widely used lipid-lowering therapies and encourage appropriate and judicious use of lipid-altering therapy in certain subpopulations. Individual monitoring in patients with risk factors, such as older females or those taking concomitant medications is warranted. Finally, the results of this analysis confirm the results of a previous systematic review concluding that the addition of ezetimibe to a statin appears unlikely to increase the incidence of myopathy (6).

\section{Acknowledgments}

Funding: This study was supported by Merck Sharp \& Dohme, Corp., Whitehouse Station, NJ, USA. Sponsor involvement in study design, data collection and data analysis, manuscript preparation and/or publication decisions is described in author contributions. The authors wish to thank Martha Vollmer, MA, of Merck Sharp \& Dohme, Corp. for editorial assistance; however, she did not meet criteria for authorship. 
Author Contributions: Dr. Toth conceived, designed, or planned the study and interpreted the results, and provided substantive suggestions for revision; Dr. Morrone conceived, designed, or planned the study and interpreted the results, and provided substantive suggestions for revision; Dr. Weintraub conceived, designed, or planned the study and interpreted the results, and provided substantive suggestions for revision; Dr. Hanson conceived, designed, or planned the study and interpreted the results; wrote sections of the initial draft; and provided administrative, technical, or logistic support; Dr. Lowe conceived, designed, or planned the study and interpreted the results; wrote sections of the initial draft; and provided administrative, technical, or logistic support; Mr. Lin conceived, designed, or planned the study, collected or assembled the data, performed or supervised analyses, and interpreted the data, provided statistical expertise, and provided substantive suggestions for revision; Dr. Shah conceived, designed, or planned the study, performed or supervised analyses, interpreted the results, provided statistical expertise, and provided substantive suggestions for revision; Dr. Tershakovec conceived, designed, or planned the study and interpreted the results, and provided substantive suggestions for revision. All authors critically reviewed subsequent iterations of the manuscript and reviewed and approved the final version of the manuscript.

\section{References}

1 Grundy SM, Cleeman JI, Merz CN et al. Implications of recent clinical trials for the National Cholesterol Education Program Adult Treatment Panel III guidelines. Circulation 2004; 110: 227-39.

2 Davidson MH, Robinson JG. Safety of aggressive lipid management. J Am Coll Cardiol 2007; 49: 1753-62.

3 Hou R, Goldberg AC. Lowering low-density lipoprotein cholesterol: statins, ezetimibe, bile acid sequestrants, and combinations: comparative efficacy and safety. Endocrinol Metab Clin North Am 2009; 38: 79-97.

4 Montecucco F, Quercioli A, Mach F. Ezetimibe/simvastatin. Expert Opin Drug Saf 2009; 8: 715 25.

5 Abd TT, Jacobson TA. Statin-induced myopathy: a review and update. Expert Opin Drug Saf 2011; 10: 373-87.

6 Slim H, Thompson PD. Ezetimibe-related myopathy: A systematic review. J Clin Lipidol 2008; 2: 328-34.

7 AstraZeneca. Crestor (Rosuvastatin Calcium) Tablets [Package Insert]. USA (Package Circular), 227. Wilmington, DE, USA: AstraZeneca, 2011.

8 Merck \& Co I. Mevacor Tablets (Lovastatin) [Package Insert]. USA(Product Circular). Whitehouse Station, NJ, USA: Merck \& Co, Inc, 2010.

9 Merck \& Co I. Zocor (Simvastatin) Tablets [Package Insert]. USA (Package Circular). Whitehouse Station, NJ, USA: Merck \& Co. Inc, 2011.

10 Novartis. Lescol (Fluvastatin Sodium) Capsules [Package Insert]. USA (Product Circular). East Hanover, NJ, USA: Novartis, 2011.

11 Parke-Davis. Lipitor (Atorvastatin Calcium) Tablets [Package Insert]. USA. New York, NY, USA: ParkeDavis, 2009.

12 Leiter LA, Betteridge DJ, Farnier $M$ et al. Lipidaltering efficacy and safety profile of combination therapy with ezetimibe/statin vs. statin monotherapy in patients with and without diabetes: an analysis of pooled data from 27 clinical trials. Diabetes Obes Metab 2011; 13: 615-28.

13 Merck/Schering-Plough Pharmaceuticals. Vytorin (Ezetimibe/Simvastatin) [Package Insert]. USA.
North Wales, PA, USA: Merck/Schering-Plough Pharmaceuticals, 2011.

14 Merck/Schering-Plough Pharmaceuticals. Zetia (Ezetimibe) [Package Insert]. USA. North Wales, PA, USA: Merck/Schering-Plough Pharmaceuticals, 2011.

15 Pasternak RC, Smith SC Jr, Bairey-Merz CN, Grundy SM, Cleeman JI, Lenfant C. ACC/AHA/NHLBI clinical advisory on the use and safety of statins. Stroke 2002; 33: 2337-41.

16 McGinnis B, Schimmer J, Hutka K. An evaluation of alanine transaminase and creatine kinase elevations with the use of ezetimibe in an ambulatory care setting. J Clin Lipidol 2010; 4: 501-7.

17 Baigent C, Landray MJ, Reith C et al. The effects of lowering LDL cholesterol with simvastatin plus ezetimibe in patients with chronic kidney disease (Study of Heart and Renal Protection): a randomised placebo-controlled trial. Lancet 2011; 377: 2181-92.

18 Kastelein JJ, Akdim F, Stroes ES et al. Simvastatin with or without ezetimibe in familial hypercholesterolemia. N Engl J Med 2008; 358: 1431-43.

19 Peto R, Emberson J, Landray M et al. Analyses of cancer data from three ezetimibe trials. $N$ Engl $J$ Med 2008; 359: 1357-66.

20 Rossebo AB, Pedersen TR, Boman $\mathrm{K}$ et al. Intensive lipid lowering with simvastatin and ezetimibe in aortic stenosis. N Engl J Med 2008; 359: 1343-56.

21 Ballantyne CM, Weiss R, Moccetti T et al. Efficacy and safety of rosuvastatin $40 \mathrm{mg}$ alone or in combination with ezetimibe in patients at high risk of cardiovascular disease (results from the EXPLORER study). Am J Cardiol 2007; 99: 673-80.

22 Bays HE, Davidson MH, Massaad R et al. Safety and efficacy of ezetimibe added on to rosuvastatin 5 or $10 \mathrm{mg}$ versus up-titration of rosuvastatin in patients with hypercholesterolemia (the ACTE Study). Am J Cardiol 2011; 108: 523-30.

23 Stein EA, Ose L, Retterstol K et al. Further reduction of low-density lipoprotein cholesterol and Creactive protein with the addition of ezetimibe to maximum-dose rosuvastatin in patients with severe hypercholesterolemia. J Clin Lipidol 2007; 1: 280-6.

24 Sudhop T, Lutjohann D, Reber M et al. Inhibition of intestinal cholesterol absorption and endogenous cholesterol synthesis by ezetimibe/simvastatin in humans [abstract]. I Am Coll Cardiol 2005; 45: 392A.

25 Davidson MH, McGarry T, Bettis R et al. Ezetimibe coadministered with simvastatin in patients with primary hypercholesterolemia. J Am Coll Cardiol 2002; 40: 2125-34.

26 Gaudiani LM, Lewin A, Meneghini L et al. Efficacy and safety of ezetimibe co-administered with simvastatin in thiazolidinedione-treated type 2 diabetic patients. Diabetes Obes Metab 2005; 7: 88-97.

27 Feldman T, Koren M, Insull W Jr et al. Treatment of high-risk patients with ezetimibe plus simvastatin co-administration versus simvastatin alone to attain National Cholesterol Education Program Adult Treatment Panel III low-density lipoprotein cholesterol goals. Am J Cardiol 2004; 93: 1481-6.

28 Ballantyne CM, Blazing MA, King TR, Brady WE, Palmisano J. Efficacy and safety of ezetimibe coadministered with simvastatin compared with atorvastatin in adults with hypercholesterolemia. Am J Cardiol 2004; 93: 1487-94.

29 Stein E, Stender S, Mata P et al. Achieving lipoprotein goals in patients at high risk with severe hypercholesterolemia: efficacy and safety of ezetimibe coadministered with atorvastatin. Am Heart J 2004; 148: 447-55.

30 Bays HE, Ose L, Fraser $\mathrm{N}$ et al. A multicenter, randomized, double-blind, placebo-controlled, factorial design study to evaluate the lipid-altering efficacy and safety profile of the ezetimibe/simvastatin tablet compared with ezetimibe and simvastatin monotherapy in patients with primary hypercholesterolemia. Clin Ther 2004; 26: 1758-73.

31 Pearson TA, Denke MA, McBride PE, Battisti WP, Brady WE, Palmisano J. A community-based, randomized trial of ezetimibe added to statin therapy to attain NCEP ATP III goals for LDL cholesterol in hypercholesterolemic patients: the ezetimibe addon to statin for effectiveness (EASE) trial. Mayo Clin Proc 2005; 80: 587-95.

32 Ballantyne CM, Abate N, Yuan Z, King TR, Palmisano J. Dose-comparison study of the combination of ezetimibe and simvastatin (Vytorin) versus atorvastatin in patients with hypercholesterolemia: the Vytorin Versus Atorvastatin (VYVA) study. Am Heart J 2005; 149: 464-73. 
33 Catapano AL, Davidson MH, Ballantyne CM et al. Lipid-altering efficacy of the ezetimibe/simvastatin single tablet versus rosuvastatin in hypercholesterolemic patients. Curr Med Res Opin 2006; 22: 204153.

34 Goldberg RB, Guyton JR, Mazzone T et al. Ezetimibe/simvastatin vs. atorvastatin in patients with type 2 diabetes mellitus and hypercholesterolemia: the VYTAL study. Mayo Clin Proc 2006; 81: 157988.

35 Conard SE, Bays HE, Leiter LA et al. Efficacy and safety of ezetimibe added on to atorvastatin (20 mg) versus uptitration of atorvastatin (to $40 \mathrm{mg}$ ) in hypercholesterolemic patients at moderately high risk for coronary heart disease. Am J Cardiol 2008; 102: 1489-94.

36 Leiter LA, Bays H, Conard S et al. Efficacy and safety of ezetimibe added on to atorvastatin (40 mg) compared with uptitration of atorvastatin (to $80 \mathrm{mg}$ ) in hypercholesterolemic patients at high risk of coronary heart disease. Am J Cardiol 2008; 102: 1495-501.

37 Robinson JG, Ballantyne CM, Grundy SM et al. Lipid-altering efficacy and safety of ezetimibe/simvastatin versus atorvastatin in patients with hypercholesterolemia and the metabolic syndrome (from the VYMET study). Am J Cardiol 2009; 103: 1694702.

38 Zieve F, Wenger NK, Ben Yehuda O et al. Safety and efficacy of ezetimibe added to atorvastatin versus up titration of atorvastatin to $40 \mathrm{mg}$ in Patients $>$ or $=65$ years of age (from the ZETia in the
ELDerly [ZETELD] study). Am J Cardiol 2010; 105: 656-63.

39 Kerzner B, Corbelli J, Sharp S et al. Efficacy and safety of ezetimibe coadministered with lovastatin in primary hypercholesterolemia. Am J Cardiol 2003; 91: 418-24.

40 Melani L, Mills R, Hassman D et al. Efficacy and safety of ezetimibe coadministered with pravastatin in patients with primary hypercholesterolemia: a prospective, randomized, double-blind trial. Eur Heart J 2003; 24: 717-28.

41 Ballantyne CM, Houri J, Notarbartolo A et al. Effect of ezetimibe coadministered with atorvastatin in 628 patients with primary hypercholesterolemia: a prospective, randomized, double-blind trial. Circulation 2003; 107: 2409-15.

42 Dobs AS, Guyton JR, McClusky D et al. Coadministration of ezetimibe with simvastatin. $\mathrm{J} \mathrm{Am}$ Coll Cardiol 2003; 41: 227A.

43 Brohet C, Banai S, Alings AM, Massaad R, Davies MJ, Allen C. LDL-C goal attainment with the addition of ezetimibe to ongoing simvastatin treatment in coronary heart disease patients with hypercholesterolemia. Curr Med Res Opin 2005; 21: 571-8.

44 Farnier M, Volpe M, Massaad R, Davies MJ, Allen C. Effect of co-administering ezetimibe with on-going simvastatin treatment on LDL-C goal attainment in hypercholesterolemic patients with coronary heart disease. Int $J$ Cardiol 2005; 102 327-32.

45 Cruz-Fernandez JM, Bedarida GV, Adgey J, Allen C, Johnson-Levonas AO, Massaad R. Efficacy and safety of ezetimibe co-administered with ongoing atorvastatin therapy in achieving low-density lipoprotein goal in patients with hypercholesterolemia and coronary heart disease. Int J Clin Pract 2005; 59: 619-27.

46 Barrios V, Amabile N, Paganelli F et al. Lipid-altering efficacy of switching from atorvastatin $10 \mathrm{mg} /$ day to ezetimibe/simvastatin $10 / 20 \mathrm{mg} /$ day compared to doubling the dose of atorvastatin in hypercholesterolaemic patients with atherosclerosis or coronary heart disease. Int J Clin Pract 2005; 59: 1377-86.

47 Constance C, Westphal S, Chung $\mathrm{N}$ et al. Efficacy of ezetimibe/simvastatin $10 / 20$ and $10 / 40 \mathrm{mg}$ compared with atorvastatin $20 \mathrm{mg}$ in patients with type 2 diabetes mellitus. Diabetes Obes Metab 2007; 9: 575-84.

48 Farnier M, Averna M, Missault L et al. Lipid-altering efficacy of ezetimibe/simvastatin $10 / 20 \mathrm{mg}$ compared with rosuvastatin $10 \mathrm{mg}$ in high-risk hypercholesterolaemic patients inadequately controlled with prior statin monotherapy - The IN-CROSS study. Int J Clin Pract 2009; 63: 547-59.

49 Gagne C, Bays HE, Weiss SR et al. Efficacy and safety of ezetimibe added to ongoing statin therapy for treatment of patients with primary hypercholesterolemia. Am J Cardiol 2002; 90: 1084-91.

50 Rodney RA, Sugimoto D, Wagman B et al. Efficacy and safety of coadministration of ezetimibe and simvastatin in African-American patients with primary hypercholesterolemia. J Natl Med Assoc 2006; 98: $772-8$.

Paper received February 2012, accepted April 2012 